\title{
Absolute calibration of vacuum ultraviolet spectrograph system for plasma diagnostics
}

\author{
M. Yoshikawa, ${ }^{\text {a) }}$ Y. Kubota, T. Kobayashi, M. Saito, N. Numada, \\ Y. Nakashima, and T. Cho \\ Plasma Research Center, University of Tsukuba, Tsukuba, Ibaraki 305-8577, Japan \\ H. Koguchi and Y. Yagi \\ National Institute of Advanced Industrial Science and Technology, Tsukuba, Ibaraki 305-8568, Japan \\ N. Yamaguchi \\ Toyota Technological Institute, Tempaku, Nagoya, Aichi 468-8511, Japan
}

(Presented on 21 April 2004; published 12 October 2004)

\begin{abstract}
A space- and time-resolving vacuum ultraviolet (VUV) spectrograph system has been applied to diagnose impurity ions behavior in plasmas produced in the tandem mirror GAMMA 10 and the reversed field pinch TPE-RX. We have carried out ray tracing calculations for obtaining the characteristics of the VUV spectrograph and calibration experiments to measure the absolute sensitivities of the VUV spectrograph system for the wavelength range from 100 to $1100 \AA$. By changing the incident angle, $50.6^{\circ}-51.4^{\circ}$, to the spectrograph whose nominal incident angle is $51^{\circ}$, we can change the observing spectral range of the VUV spectrograph. In this article, we show the ray tracing calculation results and absolute sensitivities when the angle of incidence into the VUV spectrograph is changed, and the results of VUV spectroscopic measurement in both GAMMA 10 and TPE-RX plasmas. (C) 2004 American Institute of Physics. [DOI: 10.1063/1.1789264]
\end{abstract}

\section{INTRODUCTION}

Spectroscopic measurements give us spatial and temporal variations of spectral intensity of radiation from plasmas in the wavelength ranges from visible to soft x-ray. These are the key diagnostics to determine radiation power losses and impurity ion density profiles, which directly relate to impurity transport, confinement, and sources in magnetically confined plasmas. ${ }^{1-5}$ We have developed a space- and timeresolving vacuum ultraviolet (VUV) spectrograph system, ${ }^{1,2}$ that is applied to diagnose impurity ions behavior in plasmas produced in the tandem mirror GAMMA 10 and the reversed field pinch TPE-RX.${ }^{6,7}$ For quantitative analysis of emission lines from the plasmas, it is important to characterize the absolute sensitivity of the spectrograph system throughout its wavelength range. We have carried out ray tracing calculations for obtaining characteristics of the VUV spectrograph and calibration experiments to measure the absolute sensitivities of VUV spectrograph system for the wavelength range from 100 to $1100 \AA$ using a synchrotron at the Photon Factory in the High Energy Accelerator Research Organization (KEK). By changing the incident angle, $50.6^{\circ}-51.4^{\circ}$ to the VUV spectrograph whose nominal incident angle is $51^{\circ}$, we can change the observing spectral range of the VUV spectrograph. Absolute sensitivity measurements of the VUV spectrograph with changing the angle of incidence have never been carried out. Therefore it is necessary to perform that measurement. It is useful to observe the output spectra by changing the observing wavelength range of the VUV spectrograph in this wavelength range for plasma diagnos-

${ }^{a)}$ Electronic mail: yosikawa@prc.tsukuba.ac.jp tics. This article describes the ray tracing calculation of the VUV spectrograph and measured results on the absolute sensitivities when we changed the angle of incidence for the VUV spectrograph. In GAMMA 10 and TPE-RX plasmas, there are many impurity ion emissions in this wavelength range. The impurity spectra in GAMMA 10 and TPE-RX plasmas have been measured by using this VUV spectrograph.

\section{VUV SPECTROGRAPH}

Figure 1 shows the schematic of the VUV spectrograph. The pressure inside the spectrograph is kept below 1 $\times 10^{-7}$ Torr by using two turbomolecular pumps. The VUV spectrograph can provide spatial and spectral distributions of plasma radiation in the wavelength range $150-1050 \AA$ with normal incidence configuration. It consists of an entrance slit of limited height $(100 \mu \mathrm{m} \times 2 \mathrm{~mm})$, an aberration-corrected concave grating with varied spacing grooves (Hitachi $\mathrm{P} /$ N001-0464) which gives a flat-field spectral output plane, and an image-intensified two-dimensional detector system. The specifications of the grating are that a radius of curvature is $500 \mathrm{~mm}$, nominal groove density 1200 groove $/ \mathrm{mm}$, braze angle $3.08^{\circ}$, and ruled area $48 \times 48 \mathrm{~mm}^{2}$, the nominal incident angle $51^{\circ}$, and the effective braze wavelength $600 \AA$. One can observe the upper half of the plasma with a field of view of about $25 \mathrm{~cm}$ diameter in GAMMA 10, while one can observe the center chord of the plasma with a field of view of about $18 \mathrm{~cm}$ in TPE-RX. The detector system consists of a microchannel-plate intensified image detector assembly (Hamamatsu F2814-23P, $50 \times 50 \mathrm{~mm}^{2}$ ) and an imagerecording camera. It is a high-speed solid-state camera (Reti- 


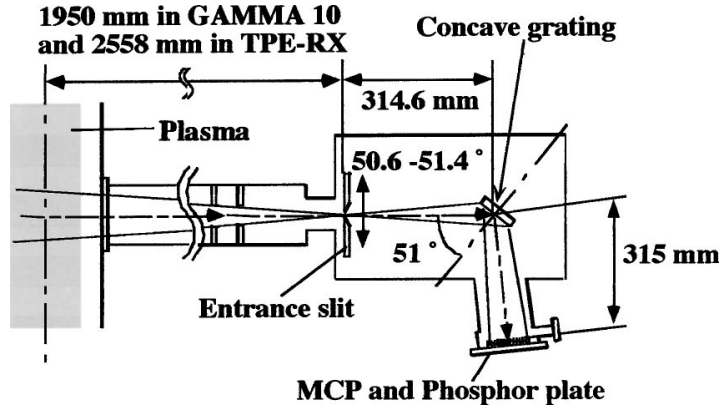

FIG. 1. Schematic view of the VUV spectrograph.

con MC9256) with a fast scanning controller. The frame rate with full image size, $256 \times 256$ pixels, can be changed from 4 to $106 \mathrm{frame} / \mathrm{s}$. Sequential space-resolving spectra can be obtained in a single plasma shot with a temporal resolution.

\section{RAY TRACINGS AND ABSOLUTE CALIBRATION EXPERIMENT}

Detailed ray tracings have been performed to understand the spectrograph characteristics, especially that of spatial imaging in the practical geometric configuration. ${ }^{1}$ The procedure of ray tracing is as follows: A ray starting from a point source goes through a point on the opening of the entrance slit and incidents on the grating at a point. The incident cosines are given from the incident ray angle and the direction cosines of the diffracted ray are then calculated by using the light path function and Fermat's principle. Finally the image point can be obtained on the output focal plane. The line source is subdivided into ten point sources and the opening of the entrance slit is divided into more than $7 \times 7$ points. After scanning rays for each point source and entrance slit point, we obtained a set of image points that shows the spectral image of the line source. Ray tracings have been performed for an entrance slit $100 \mu \mathrm{m}$ wide and $2 \mathrm{~mm}$ high, and wavelengths in the region from 150 to $1100 \AA$ by changing the incident angle, $50.6^{\circ}, 51^{\circ}$, and $51.4^{\circ}$. Figures 2(a)-2(c) show the results of ray tracings corresponding to incident angles of $50.6^{\circ}, 51^{\circ}$, and $51.4^{\circ}$, respectively, where five line sources placed $5 \mathrm{~cm}$ apart are assumed. We can make mapping the source position at the plasma on the detector plane from these results. The spatial resolution is esti-

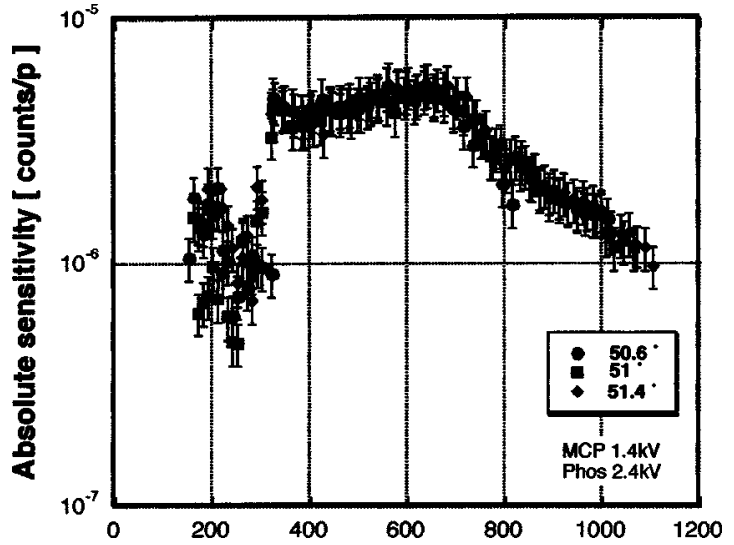

FIG. 3. Absolute sensitivity of the VUV spectrograph for the first order diffracted light as a function of wavelength. Circles, squares, and diamonds show the absolute sensitivities of incident angle of $50.6^{\circ}, 51^{\circ}$, and $51.4^{\circ}$, respectively.

mated to be $4.2-4.4 \mathrm{~mm}$. The spectral resolution is $2 \AA$ over the full spectral range at the incident angle of $51^{\circ}$. When we set the incident angles of $50.6^{\circ}$ and $51.4^{\circ}$, the spatial resolutions are almost the same as that of $51^{\circ}$ and the spectral resolutions are about $6 \AA$, which is three times as bad as that of $51^{\circ}$.

Absolute calibration experiments for the VUV spectrograph have been performed by using the synchrotron radiation at PF in KEK (BL-11C and BL-12A). The incident photon intensity was monitored just behind the entrance slit by using an absolutely calibrated extreme ultraviolet silicon photodiode (IRD AXUV 100) and the output spectral image was recorded on a high-speed camera. Measurements were repeated for incident wavelength from 50 to $350 \AA$ with intervals of $10 \AA$ at BL-12A and from 300 to $1100 \AA$ with intervals of $20 \AA$ at BL-11C under the incident angles of $50.6^{\circ}, 51^{\circ}$, and $51.4^{\circ}$. Recorded images were directly analyzed by using a personal computer. Figure 3 shows an absolute sensitivity of the VUV spectrograph for the first order diffracted light as a function of wavelength. Circles, squares, and diamonds show the absolute sensitivities of incident angles of $50.6^{\circ}, 51^{\circ}$, or $51.4^{\circ}$, respectively. The position of a peak (at $600 \AA$ ) corresponds to the braze wavelength of the grating used in this spectrograph. By changing the incident angle, $50.6^{\circ}, 51^{\circ}$, and $51.4^{\circ}$, the observable spectral range of (a)

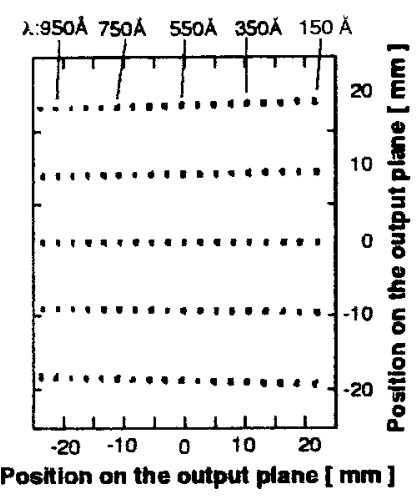

(b)

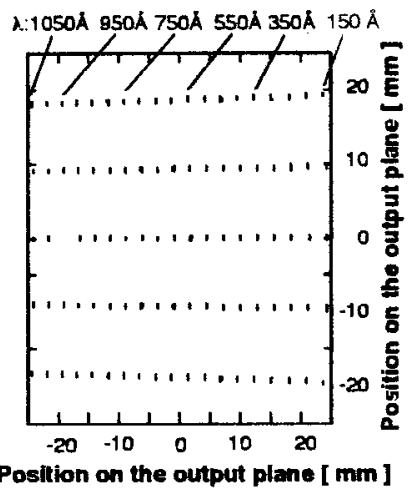

(c)

X:1050A $950 \AA 750 \AA 550 \AA 350 \AA 200 \AA$

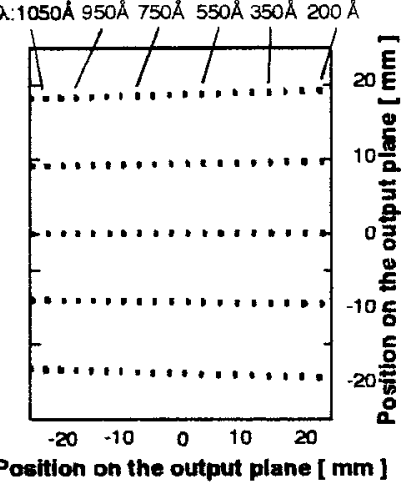

FIG. 2. Results of ray tracings of incident angle of (a) $50.6^{\circ}$, (b) $51^{\circ}$, and (c) $51.4^{\circ}$, respectively, where five line sources placed $5 \mathrm{~cm}$ apart are assumed. 
the VUV spectrograph is changed, 150-1020, 160-1060, and $170-1090 \AA$, respectively. The sensitivity against incident angle variation is almost the same as that of the nominal incident angle of $51^{\circ}$.

\section{PLASMA SPECTROSCOPY}

We used the VUV spectrograph for plasma diagnostics in both GAMMA 10 and TPE-RX. In the tandem mirror GAMMA 10, plasma confinement is achieved by not only a magnetic mirror configuration but also high potentials at both end regions. The potentials are produced by means of electron cyclotron resonance heating at the plug/barrier region. The main plasma confined in GAMMA 10 is produced and heated by the ion cyclotron range of frequency power deposition. The electron density, electron temperature, and ion temperature are about $2 \times 10^{12} \mathrm{~cm}^{-3}, 80 \mathrm{eV}$, and $5 \mathrm{keV}$, respectively. ${ }^{6}$ The characteristics of the reversed field pinch plasma device TPE-RX are $R / a=1.72 / 0.45 \mathrm{~m}$, where $R$ and $a$ are major and minor radii, respectively. ${ }^{7}$ The vacuum vessel is made of stainless steel and the inner limiter made of molybdenum is installed. The designed maximum plasma current $I_{p}$ and the pulse duration time are $1 \mathrm{MA}$ and $100 \mathrm{~ms}$, respectively. The experimental conditions used here are as follows: $I_{p}=300 \mathrm{kA}$, the line averaged density as determined by an interferometer is in the range of $5-25 \times 10^{12} \mathrm{~cm}^{-3}$, which almost linearly scales with $I_{p}$, and the electron temperature determined by the Thomson scattering system is in the range of $100-500 \mathrm{eV}$.

We checked the observing spectral range of the VUV spectrograph by changing the incident angle of $51^{\circ}$ in GAMMA 10 and 50.6 in TPE-RX plasma. Figure 4(a) shows the impurity line spectrum at the center of the GAMMA 10 plasma with the VUV spectrograph incident angle of $51^{\circ}$, while Fig. 4(b) shows that of the TPE-RX plasma with the VUV spectrograph incident angle of $50.6^{\circ}$. Most all of the spectral lines are identified as emission lines of carbon and oxygen ions. In GAMMA 10, there are small background emissions. However, the spectrum in TPE-RX shows the strong background emissions. There are many impurity ion emissions of $\mathrm{Fe}, \mathrm{Cr}, \mathrm{Ni}, \mathrm{Mo}$, and/or $\mathrm{Cu}$, which are the structures in the TPE-RX device. Emission lines from higher- $Z$ impurity ions are so crowded that they overlap each other, and often appear as quasicontinuum spectra. The difference in appearance of the spectra between Figs. 4(a) and 4(b) comes from these higher- $Z$ impurity ion line emissions. From these experiments we certify the spectral range of VUV spectrograph by changing the incident angles. The spectral resolutions for the two incident angles are almost the same, which is expected by the ray tracing calculation.
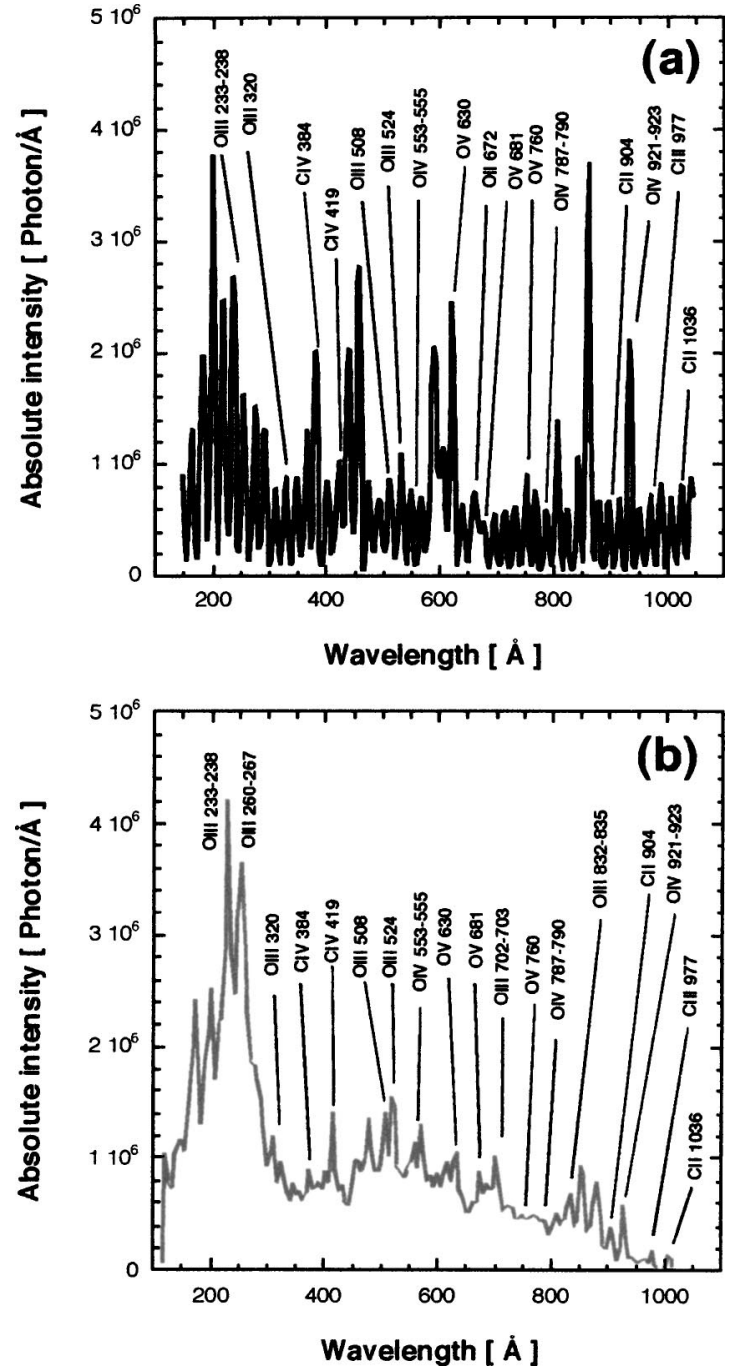

FIG. 4. (a) Impurity line spectrum at the center of the GAMMA 10 plasma with the VUY spectrograph incident angle of $51^{\circ}$ and (b) TPE-RX plasma with the VUV spectrograph incident angle of $50.6^{\circ}$.

\section{ACKNOWLEDGMENTS}

The authors would like to thank members of GAMMA 10 group of the University of Tsukuba and TPE-RX group of National Institute of Advanced Industrial Science and Technology for their collaboration. Part of this work was supported by a University of Tsukuba Project Research Fund. Part of the work has been performed under the approval of the Photon Factory Advisory Committee.

\footnotetext{
${ }^{1}$ N. Yamaguchi et al., Rev. Sci. Instrum. 65, 3408 (1994).

${ }^{2}$ M. Yoshikawa et al., Surf. Rev. Lett. 9, 555 (2002).

${ }^{3}$ M. Yoshikawa et al., Trans. Fusion Technol. 43, 189 (2003).

${ }^{4}$ T. Kato et al., Fusion Eng. Des. 34-35, 25 (1997).

${ }^{5}$ S. Morita et al., Rev. Sci. Instrum. 74, 2036 (2003).

${ }^{6}$ K. Yatsu et al., Trans. Fusion Sci. Technol. 43, 10 (2003)

${ }^{7}$ Y. Yagi, Nucl. Fusion 40, 1933 (2000).
} 